\title{
Ulcerated tophaceous gout
}

\author{
Michelle Gita Filanovsky, ${ }_{1}^{1}$ Kumar Sukhdeo, ${ }^{2}$ Megan Cunnane McNamara ${ }^{3}$
}

${ }^{1}$ Case Western Reserve University School of Medicine, Cleveland, Ohio, USA

${ }^{2}$ Case Western Reserve University, Cleveland, Ohio, USA

${ }^{3}$ Department of Internal Medicine, Louis Stokes Veterans Administration Medical Center, Cleveland, Ohio, USA

\section{Correspondence to} Dr Kumar Sukhdeo, sukhdeo@gmail.com

Accepted 11 July 2015

\section{CrossMark}

To cite: Filanovsky MG, Sukhdeo K, McNamara MC. BMJ Case Rep Published online: [please include Day Month Year] doi:10.1136/ bcr-2015-210707

\section{SUMMARY}

Gout is an inflammatory arthritis characterised by hyperuricemia, which, if poorly controlled, can lead to the development of tophi. We report the case of a 60-year-old Caucasian man with poorly controlled polyarticular tophaceous gout with multiple comorbidities (including renal failure) who presented with tophaceous ulcers of the upper extremity. These ulcers caused extreme pain, requiring chronic opiate medications, and were associated with decreased sensation and reduced ability to move the extremity. His hospital course was complicated by acute kidney injury, haemolytic anaemia and Clostridium difficile infection. He required 1 month of antibiotics and intensive wound care for his ulcers. This case highlights the diagnosis, natural history and management of an unusual complication of hyperuricemia.

\section{BACKGROUND}

Gout is an inflammatory arthritis characterised by hyperuricemia, and deposition of monosodium urate crystals within joints and other tissues when uric acid levels exceed $6.8 \mathrm{mg} / \mathrm{dL}^{1}{ }^{1}$ These acute 'gout attacks' cause an exquisitely painful monoarticular arthritis, which resolves with treatment. In between attacks, patients are typically asymptomatic. However, with inadequate control of hyperuricemia, up to $30 \%$ of patients with gout develop chronic deposition of crystals, a condition termed tophaceous gout. ${ }^{12}$ These patients may experience chronic pain even between acute attacks, and often have polyarticular involvement. Tophi typically occur within or around joints, but can also develop on tendons, subcutaneously (sometimes causing bullae), and in rare areas such as the heart valves and cornea. ${ }^{3-8}$ Despite the fact that tophi can become relatively large and cause joint deformities, ulceration of the skin by tophi is very uncommon and extremely difficult to treat, with no set guidelines regarding treatment. Our literature search yielded only four case reports representing seven patients with ulcerative gout. ${ }^{9-12}$ We report a case of poorly controlled polyarticular tophaceous gout complicated by tophaceous ulcers and associated cellulitis. We report this case in order to contribute to the limited knowledge of this rare disease process present in the literature.

\section{CASE PRESENTATION}

A 60-year-old Caucasian man with a long-standing history of poorly controlled polyarticular tophaceous gout presented with a swollen, erythematous, extremely painful left forearm and hand. The patient also had numbness and tingling, as well as difficulty moving the extremity. His gout had been very poorly controlled, as he was non-compliant with his therapeutic regimen, which included colchicine $0.6 \mathrm{mg}$ every other day and febuxostat $40 \mathrm{mg}$ every day. He had approximately one emergency department visit per month for recurrent gout flairs and cellulitis (possibly related to superinfection of subcutaneous tophi, although this is unclear) during the 6 months leading up to this admission, requiring multiple prednisone tapers and antibiotic regimens. His other medical history included stage 3 chronic kidney disease, type II diabetes mellitus complicated by neuropathy, hypertension, hypothyroidism, chronic venous insufficiency, atrial fibrillation and anxiety. Medications in addition to the gout therapies listed above included diltiazem, furosemide, gabapentin, hydroxyzine, insulin, levothyroxine, metoprolol, omeprazole, warfarin and morphine sustained-release tablets (for pain associated with gout). He was a former smoker with a 10 pack-year history, and he had a prior history of alcohol and cocaine abuse.

On admission, vital signs were normal except for tachycardia reaching $130 \mathrm{bpm}$. On examination, his left upper extremity appeared diffusely warm and erythematous with three haemorrhagic bullae on the ventral forearm and palm; no crepitus was present. Pulses were not palpable in the left upper extremity. In addition, the patient was noted to have multiple subcutaneous $1-3 \mathrm{~mm}$ pustule-like tophi on the palm of the right hand, as well as palpable tophi in most of the joints of the bilateral hands, and bilateral dorsal and ventral forearms. Laboratory tests were significant for leucocytosis $\left(34.4 \times 10^{9}\right.$ cells $\left./ \mu \mathrm{L}\right)$, elevated creatinine from his baseline $(2.7 \mathrm{mg} / \mathrm{dL})$, hyperuricemia $(12.4 \mathrm{mg} / \mathrm{dL})$ and an erythrocyte sedimentation rate of $32 \mathrm{~mm} / \mathrm{h}$.

The patient thus met two systemic inflammatory response syndrome criteria, and was started on intravenous vancomycin and ceftriaxone for presumed sepsis. Blood cultures showed no growth, however, wound cultures grew oxacillin-resistant Staphylococcus hominis. With antibiotics and intravenous fluids, the patient's acute kidney injury and leucocytosis resolved. In addition, his arm became less erythematous and the bullae ruptured (figures 1 and 2). However, the patient still continued to have severe pain, numbness and difficulty moving the extremity. He required opiate medications for pain control. His hospital course was complicated by the development of a Clostridium difficile infection, requiring oral metronidazole. His left upper extremity wounds were treated with silver sulfadiazine medicated wraps. Nine days after admission, he was discharged to a nursing facility for continued antibiotics and wound care. 


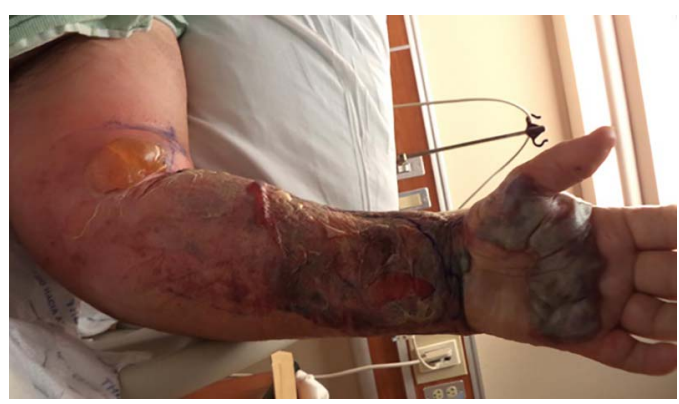

Figure 1 Left upper extremity on day 5 of first admission, illustrating continued erythema and haemorrhagic bullae.

While at the nursing facility, he continued to receive antibiotics for cellulitis and C. difficile infection, for a total regimen of 2 weeks of intravenous ceftriaxone and vancomycin, followed by 2 weeks of intravenous ceftaroline and oral metronidazole. As the roofs of the bullae were removed, ulcers containing tophi were more clearly revealed on the left ventral forearm, as well as on the palm and in the interdigital area between the thumb and first finger (figure 3). While at the nursing facility, the wound care regimen was switched to AQUACEL Silver. The patient continued to require opiates for pain control.

Thirty-seven days after initial admission, the patient was noted to have increased erythema of the left upper extremity as well increased pain. Thus, the patient was readmitted to the hospital due to concerns for recurrent cellulitis. On admission, vital signs were normal except for hypotension (102/ $72 \mathrm{~mm} \mathrm{Hg}$ ). On examination, his left upper extremity appeared slightly erythematous with tophaceous ulcers in the same locations noted above, however, the ulcers were smaller in size; in addition, the two ulcers on the ventral forearm seemed to be communicating subcutaneously and probed to deep musculature (figure 4). A thick, yellow exudate was expressed from all the ulcers. Laboratory tests were significant for elevated creatinine from baseline $(3 \mathrm{mg} / \mathrm{dL}$ ), anaemia (haemoglobin $8.3 \mathrm{~g} / \mathrm{dL}$, mean corpuscular volume $90 \mathrm{fL}, 3.68 \%$ reticulocytes, haptoglobin $<6 \mathrm{mg} / \mathrm{dL}$, lactate dehydrogenase $237 \mathrm{U} / \mathrm{L})$, hyperuricemia $(10.3 \mathrm{mg} / \mathrm{dL})$, erythrocyte sedimentation rate $111 \mathrm{~mm} / \mathrm{h}$ and $\mathrm{C}$ reactive protein $5.8 \mathrm{mg} / \mathrm{dL}$. Blood cultures exhibited no growth, and wound cultures grew skin flora (S. epidermis). Subcutaneous emphysema was present on X-ray of the left upper extremity; however, no crepitus or intense spreading erythema was present, and air was likely present due to air trapping through chronic ulcers. On the basis of above findings, there was low concern for cellulitis or necrotising fasciitis.

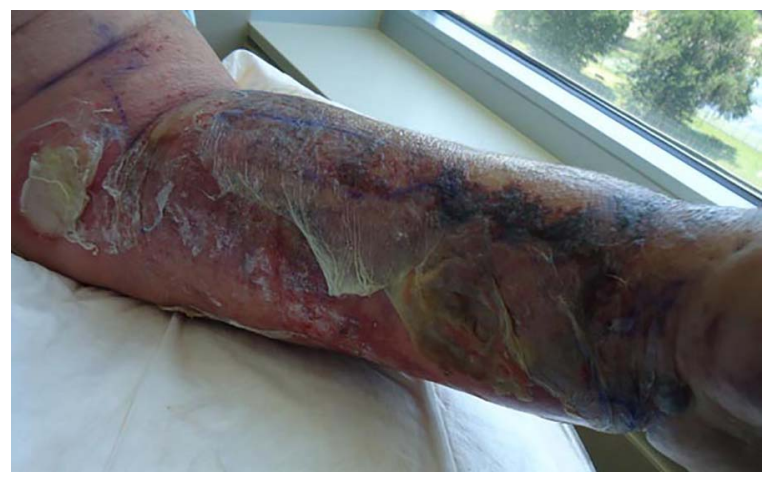

Figure 2 On the day of discharge from first admission (day 9). Bullae are resolving.
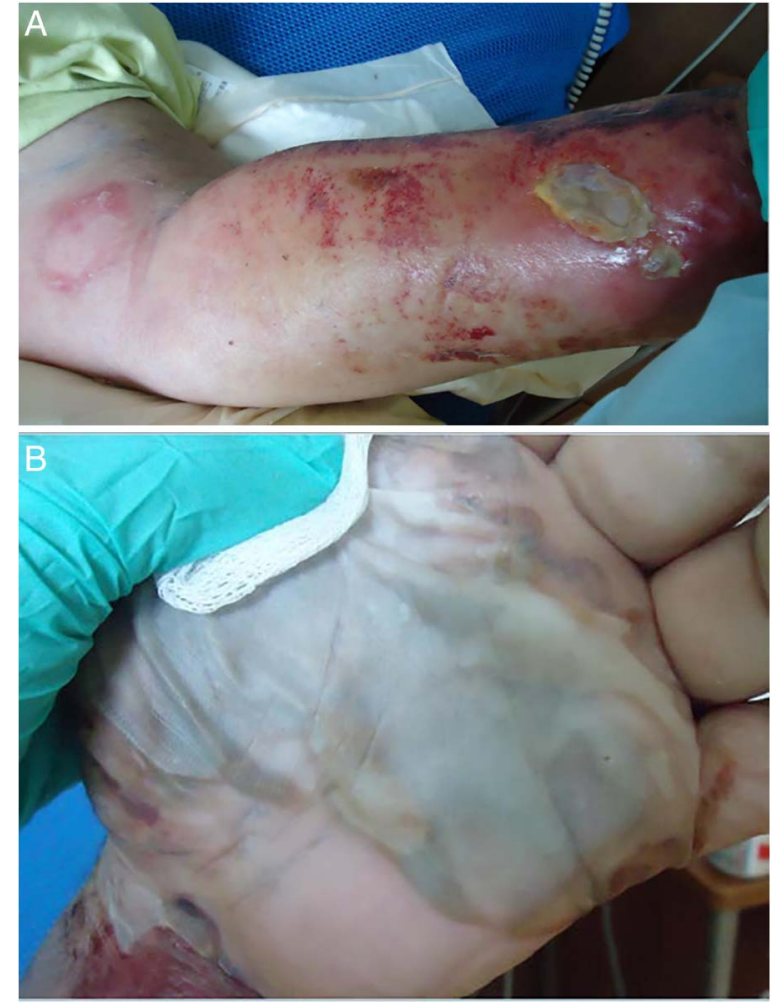

Figure 3 (A) Three days after discharge (12 days after admission). Bulla roof has been removed, revealing underlying tophaceous ulcers on the ventral forearm. (B) Three days after discharge (12 days after admission). Bulla on palm is resolving and no longer haemorrhagic.

Acute kidney injury and blood pressure improved with the administration of intravenous fluids. The origin of the patient's haemolytic anaemia remains unknown, as a thorough work up of potential malignancy, infection and inherent red blood cell defects was negative. It is possible that haemolysis occurred due to antibiotic use, as antibiotics are a common cause of drug-induced haemolytic anaemia, especially ceftriaxone. ${ }^{13}$ The patient required a 2 unit transfusion of packed red blood cells. Haemoglobin and haptoglobin trended up, while reticulocytes and lactate dehydrogenase trended down, indicating resolution of haemolysis. The patient's hospital stay was also complicated by an acute polyarticular gout flair in his right upper extremity, requiring a 10 -day prednisone taper. During his hospital stay, his topical wound treatment regimen was changed to medicalgrade honey (MediHoney), with which the patient experienced improvement (figure 5). After 10 days of hospitalisation, the patient was discharged back to the nursing facility for further wound care.

\section{OUTCOME AND FOLLOW-UP}

At the time of submission of this report (1 month after last admission), the patient remains at the nursing facility. Ulcerations are still present and continue to be treated with medical-grade honey. He continues to have severe pain in his left upper extremity, requiring opiate medications. He also continues to have decreased sensation and difficulty moving the extremity. His kidney function and haemoglobin remain stable, and he has not required any further admissions. His gout regimen remains the same as prior, however, his rheumatology team is planning to start a uricase agent shortly. 


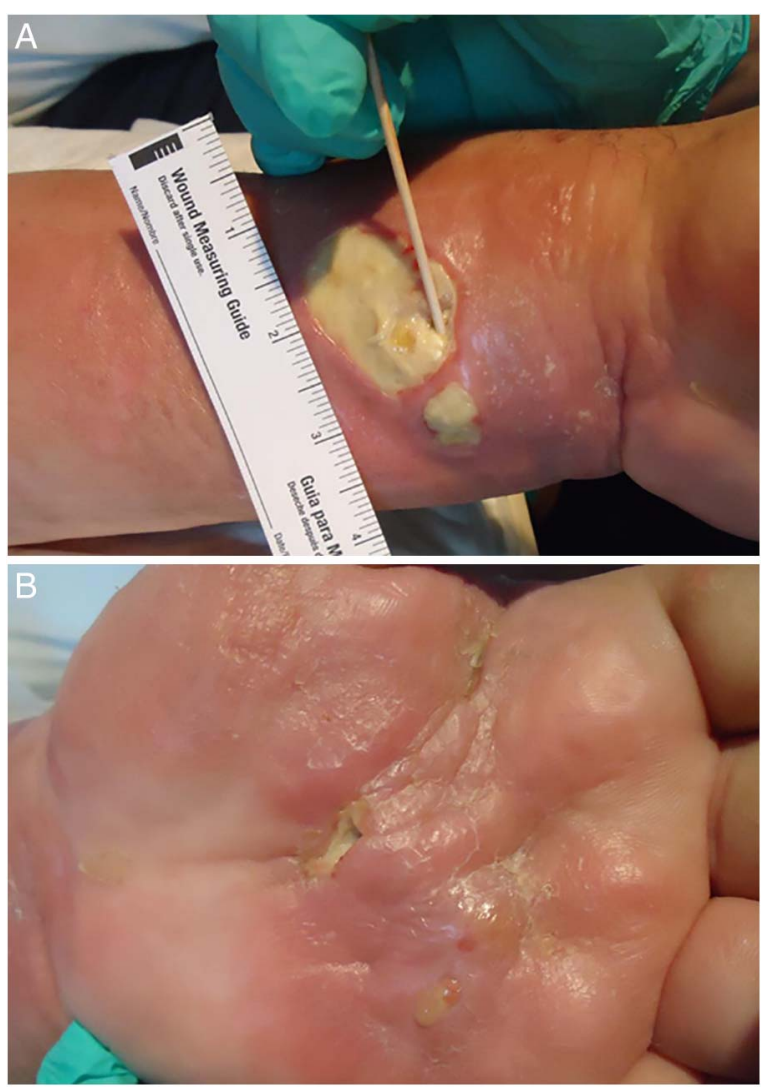

Figure 4 (A) On day of second admission (37 days after first admission). Communicating ulcers on the ventral left forearm with surrounding mild erythema. (B) On day of second admission (37 days after first admission). Tophaceous ulcer on palm.

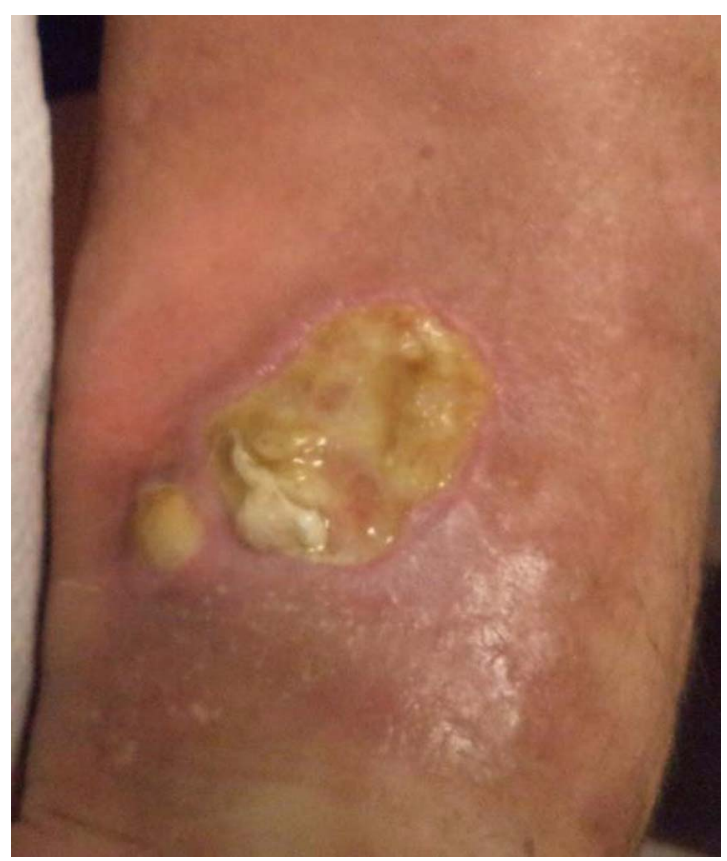

Figure 5 On day of second admission discharge (10 days after second admission, 47 days after first admission). Improved ventral forearm ulcer after beginning the use of medical-grade honey (MediHoney) 1-week earlier.

\section{DISCUSSION}

Our case illustrates a rare complication of poorly controlled gout. On the basis of our literature review, there are no investigational studies regarding tophaceous ulcers, thus little is known about their epidemiology, pathophysiology, clinical course, diagnosis and treatment. Our literature search yielded four recent case reports of ulcerative gout, representing seven patients. ${ }^{9-12}$ The patients in these reports were mostly male, with only two female patients. Ages ranged from 45 to 84 years old. Interestingly, three reported patients had no history of gout prior to presentation with tophaceous ulcers. ${ }^{9} 1112$ In addition, five patients had high uric acid levels on presentation. ${ }^{9-12}$ The great majority of ulcers were located on the lower extremities, with only two reports of patients with upper extremity tophaceous ulcers; of note, these two patients also had ulcers present on the lower extremities. ${ }^{9} 10$ While almost all prior reports discuss the pain associated with tophaceous ulcers, no other reports mention the presence of numbness and mechanical impairment of the affected extremities, as was present in our patient.

There are no reports of patients with tophaceous ulcers developing severe cellulitis with haemorrhagic bullae as did our patient, however, there are two reports of more localised tophaceous ulcer wound infections, ${ }^{10}{ }^{11}$ with one patient growing Escherichia coli from his lower extremity wound. ${ }^{11}$ It is important to note that, in our patient, it was difficult to determine whether the cellulitis caused the eruption of tophaceous ulcers, or whether tophi beginning to ulcerate caused the cellulitis. Of note, bullous tophaceous eruptions have been previously reported; ${ }^{8}$ thus, tophi may have contributed to the development of bullae in our patient.

Our case also illustrates the debilitating, chronic nature of ulcers caused by tophaceous gout. The chronicity of these lesions is likely multifactorial, with prior case reports noting ulcer healing times ranging from 8 days to over 3 years. ${ }^{10-12}$ Many patients with gout have comorbidities, such as peripheral vascular disease and diabetes, which can impair wound healing. Antihyperuricemic medications often decrease tophi size very slowly. ${ }^{14} 15$ Thus, complete resolution of tophi can take years, and they will be present as a possible source of ulceration and infection for significant periods of time.

Surgical techniques are often considered to remove or decrease the size of cutaneous tophi. General indications for surgery include infection of tophi, ulcerated tophi, mechanical impairments, uncontrollable pain and cosmesis. ${ }^{16} 17$ Classic curettage and debridement can be used to remove tophi, however, this technique has high rates of delayed wound healing and skin necrosis. ${ }^{18}$ Thus, other techniques, such as shaving and hydrosurgery, have been developed. ${ }^{17}{ }^{18}$ In addition, skin grafting can be performed. ${ }^{9}$ Surgery was never considered in our patient, however, it may be considered in the future, as he meets multiple criteria.

There are no set guidelines for wound care of tophaceous ulcers. Prior case reports demonstrated success with the use of hydrogel, silver-containing wound dressings, heterologous lyophilised collagen and citric acid ointment. ${ }^{10-12}$ Our patient's ulcers responded best to medical-grade honey (MediHoney), which has antimicrobial activity, and has been shown to accelerate wound healing and cause autolytic wound debridement. ${ }^{19}$

As was illustrated in our patient, tophaceous ulcers are not only difficult to treat, but are also extremely debilitating. Our patient has chronic pain requiring opiate medications, decreased functionality of his limb and diminished sensation in this area. The patient's diabetes mellitus likely contributed to these 
symptoms as well. He also requires multiple wound dressings per day and constant monitoring for wound infection, and thus is currently unable to return home, and is required to live in a nursing facility. A prior case series illustrated that patients with tophaceous ulcers had lower quality of life scores than patients with chronic venous leg ulcers. ${ }^{12}$

Our patient's case also illustrates the difficulty of controlling gout in those with multiple comorbidities. As in our patient, many others with gout have multiple comorbidities including obesity, diabetes, peripheral vascular disease, hypertension, chronic kidney disease and hyperlipidaemia. ${ }^{1} 2021$ Control of hyperuricemia is extremely important in the treatment of gout and prevention of tophi; however, the frequency of multiple comorbidities, especially chronic kidney disease, makes treatment difficult. The American College of Rheumatology guidelines on the treatment of gout recommend that patients first be treated with xanthine-oxidase inhibitors, followed by the addition of uricosuric and uricase medications, if necessary. ${ }^{22}$ However, many first-line medications, such as allopurinol and probenecid, are often contraindicated in patients with severe renal failure. ${ }^{1}{ }^{14}{ }^{22}$ Recently, interleukin 1 inhibitors, such as anakinra, are being investigated as potential alternative treatments for refractory gout; ${ }^{15}$ however, anakinra also requires renal dosing. Thus, patients with chronic kidney disease are often limited in terms of therapeutic options, with febuxostat and uricase agents often being used, although the safety of these drugs in severe renal failure has not been reported. ${ }^{23}$ The American College of Rheumatology Guidelines also stress the importance of behavioural modification, including weight loss, smoking cessation and decreasing intake of purine-rich foods, such as meats, seafood and alcohol. However, behavioural changes are rarely effective on their own.

In summary, this case highlights an unusual manifestation of poorly controlled gout, as well as the difficulty of controlling chronic tophaceous gout in a patient with multiple comorbidities. Although ulcerative tophi are a rare entity, they have a significant morbidity and greatly impact patients' quality of life.

Learning points

- In patients with gout, control of hyperuricemia with behavioural modification and medication is crucial to prevent the development of tophi.

- Ulcers caused by tophaceous gout are rare but extremely difficult to treat, and are very debilitating. Although there is no investigative literature on the topic, from our experience and prior case reports, treatment involves antihyperuricemic agents, intensive wound care, close monitoring for signs of infection and, possibly, surgery.

- Chronic tophaceous gout is often present with multiple comorbidities, which create a therapeutic challenge, especially renal failure. Options include febuxostat and uricase agents for patients with mild-moderate renal failure, however, it is unclear if these agents are appropriate in severe renal failure.
Further research should be aimed towards identifying the best medical, surgical and wound care regimens for the treatment of tophaceous ulcers, as well as appropriate antihyperuricemic medical regimens for patients with severe tophaceous gout and renal failure.

Contributors KS and MGF performed the literature review and wrote the initial draft of the manuscript. MGF and MCM examined and treated the patient. MCM provided mentorship and guidance in clinical reasoning and revised the manuscript.

Funding National Institutes of Health; National Cancer Institute.

Competing interests None declared.

Patient consent Obtained.

Provenance and peer review Not commissioned; externally peer reviewed.

\section{REFERENCES}

1 Neogi T. Gout. N Engl J Med 2011;364:443-52.

2 Perez-Ruiz F, Martinez-Indart L, Carmona L, et al. Tophaceous gout and high leve of hyperuricaemia are both associated with increased risk of mortality in patients with gout. Ann Rheum Dis 2013;73:177-82.

3 Apibal Y, Jirasuthus S, Puavilai S. Abruption pustular gouty tophi of palm and sole. J Med Assoc Thai 2009;92:979-82.

4 Forbess LJ, Fields TR. The broad spectrum of urate crystal deposition: unusual presentations of gouty tophi. Semin Arthritis Rheum 2012;42:146-54.

5 lacobellis $\mathrm{G}$, lacobellis $\mathrm{G}$. A rare and asymptomatic case of mitral valve tophus associated with severe gouty tophaceous arthritis. J Endocrinol Invest 2014;27:965-6.

6 Iwamoto T, Toki H, Ikari K, et al. Multiple extensor tendon ruptures caused by tophaceous gout. Mod Rheumatol 2010;20:210-12.

7 Sarma P, Das D, Deka P, et al. Subconjunctival urate crystals: a case report. Cornea 2010;29:830-2

8 Schumacher HR. Bullous tophi in gout. Ann Rheum Dis 1977;36:91-3.

9 Bassi J, Mahindra P, Singh J. Bilateral upper and lower limb ulcers due to gout-a case report. Indian J Orthop 2006:40:53-4.

10 Falidas E, Rallis E, Bournia VK, et al. Multiarticular chronic tophaceous gout with severe and multiple ulcerations: a case report. J Med Case Rep 2011;5:397.

11 Nagoba BS, Punpale A, Poddar A, et al. Citric acid treatment of chronic nonhealing ulcerated tophaceous gout with bursitis. Int J Low Extrem Wounds 2013:12:276-8.

12 Patel GK, Davies WL, Price PP, et al. Ulcerated tophaceous gout. Int Wound J 2010;7:423-7.

13 Garratty G. Immune hemolytic anemia associated with drug therapy. Blood Rev 2010;24:143-50.

14 Richette $\mathrm{P}$, Briére $\mathrm{C}$, Hoenen-Clavert $\mathrm{V}$, et al. Rasburicase for tophaceous gout not treatable with allopurinol: an exploratory study. J Rheumatol 2007;34:2093-8.

15 Tran AP, Edelman J. Interleukin-1 inhibition by anakinra in refractory chronic tophaceous gout. Int J Rheum Dis 2011;14:e33-7.

16 Kumar S, Gow P. A survey of indications, results and complications of surgery for tophaceous gout. N Z Med J 2002;115:U109.

17 Lee SS, Sun IF, Lu YM, et al. Surgical treatment of the chronic tophaceous deformity in upper extremities-the shaving technique. J Plast Reconstr Aes 2009;62:669-74.

18 Lee JH, Park JY, Seo JW, et al. Surgical treatment of subcutaneous tophaceous gout. J Plast Reconstr Aesthet Surg 2010;63:1933-5.

19 Biglari B, Moghaddam A, Santos K, et al. Multicentre prospective observational study on professional wound care using honey (MedihoneyTM). Int Wound J 2013;10:252-9.

20 Choi HK, Atkinson K, Karlson EW. Obesity, weight change, hypertension, diuretic use, and risk of gout in men: the health professionals follow-up study. Arch Intern Med 2005;165:742-8.

21 Johnson RJ, Nakagawa T, Jalal D, et al. Uric acid and chronic kidney disease: which is chasing which? Nephrol Dial Transplant 2013;28:2221-8.

22 Khanna D, Fitzgerald JD, Khanna PP, et al. 2012 American College of Rheumatology guidelines for management of gout. Part 1: systematic nonpharmacologic and pharmacologic therapeutic approaches to hyperuricemia. Arthritis Care Res 2012;64:1431-46.

23 Curiel RV, Guzman NJ. Challenges associated with the management of gouty arthritis in patients with chronic kidney disease: a systematic review. Semin Arthritis Rheum 2012;42:166-78. 
Copyright 2015 BMJ Publishing Group. All rights reserved. For permission to reuse any of this content visit http://group.bmj.com/group/rights-licensing/permissions.

BMJ Case Report Fellows may re-use this article for personal use and teaching without any further permission.

Become a Fellow of BMJ Case Reports today and you can:

- Submit as many cases as you like

- Enjoy fast sympathetic peer review and rapid publication of accepted articles

- Access all the published articles

- Re-use any of the published material for personal use and teaching without further permission

For information on Institutional Fellowships contact consortiasales@bmjgroup.com

Visit casereports.bmj.com for more articles like this and to become a Fellow 